\title{
The Strategy of Improving the Information Literacy of Minority College Students
}

\author{
Jun $\mathrm{Ma}^{1, \mathrm{a}^{*}}$ and Yongfeng $\mathrm{Ma}^{1, \mathrm{~b}}$
}

${ }^{1}$ College of Educational Science and Technology, Northwest Minzu University, Lanzhou, Gansu, 730030, China

$$
\text { a364221500@qq.com, b63960396@qq.com }
$$

*the corresponding author

Keywords: National minority; College students; Information literacy; Countermeasures

\begin{abstract}
This paper mainly studies the methods of improving the information literacy of minority college students, analyzes the information literacy of them from four aspects: information awareness, information knowledge, information ability and information ethics through sampling questionnaire survey of students in Northwest Ninzu University, finds out the gap between minority college students and other students in terms of average information literacy, and analyzes the reasons, so as to summarize the countermeasures of improving the ability of information retrieval, information processing, information identification, information evaluation, information processing, information transmission and information creation of minority college students.
\end{abstract}

Acknowledgements: This study is supported by the following projects: The basic scientific research business expense project of central universities of "a good and three distinctive" subject of Northwest Minzu University in 2017 “Northwest Ethno-National Studies"(NO:31920170096); "Research on the Application of Electronic Learning Portfolio to Promote the Teaching Effect”(NO:2014JG-2670030634).

\section{少数民族大学生信息素养提升策略 \\ 马俊 ${ }^{1, a}$, 马永峰 ${ }^{1, b}$}

西北民族大学 教育科学与技术学院，中国 甘肃 兰州 730030

a364221500@qq.com, b63960396@qq.com

摘要: 主要研究少数民族大学生提升信息素养的方法, 通过对中国西北民族大学学生抽样问卷调查, 分析其信息意识、 信息知识、信息能力、信息道德等四个方面的信息素养现状, 找出少数民族大学生在平均信息素养方面和其他学生相比较存 在的差距，分析其原因，总结出提高少数民族大学生信息检索、信息处理、信息识别、信息评价、信息加工、信息传递和信 息创造能力的对策。

关键词: 少数民族; 大学生; 信息素养; 对策

中图分类号：G750 文献标志码：A

\section{1 引言}

进入 21 世纪后，信息素养在全球范围内展开。2003 年 9 月在捷克召开的布拉格会议中，将信息素养 定义为一种能力, 它能够确定、查找、评估、组织和有效地生产、使用和交流信息, 来解决一个问题 [1]。 随着计算机网络技术的迅速发展和普及, 计算机网络被广泛应用于各个领域, 使得每天都有海量的信息在 更新。据有关调查分析：一个大学生刚刚迈出校门, 其所学的知识有近 $50 \%$ 即已过时。在这种日新月异的 信息环境中, 具备良好的信息素养对现代大学生十分为重要。尤其是民族高校的大学生, 他们是少数民族 的高素质人才，将从事我国民族理论和民族政策的研究，传承和弘扬民族优秀文化。需要找到培养他们的 
信息检索、分辨、评价和组合能力, 才能从大量却不确定的信息中提取出自己所需要的内容，提高信息的 使用能力和创新能力。通过调查分析少数民族大学生信息素养现状, 找出培养和提高他们信息素养水平的 具体方法。

\section{2 信息素养构成}

信息素养主要由信息意识（意识层面）、信息知识（知识层面）、信息能力（技术层面）以及信息伦理 道德（道德层面）等四部分构成 $[2]$ 。

\section{1 信息意识}

信息意识是人们产生获取所需信息的内在动力和原因, 是人们在信息活动中产生的认识、观念和需求 的总和。信息意识主要包括：能认识到信息素养在信息时代中信息的重要性; 对信息有积极的内在需求; 对信息的敏感性和洞察力。

\section{2 信息知识}

信息知识是指一切与信息有关的理论、知识和方法。信息知识是信息素养的重要组成部分，一般来说 包括：传统文化素养；信息的基本知识；信息的方法与原则；现代信息技术知识等 [3]。

\section{3 信息能力}

信息能力是指人们有效利用信息设备和信息资源获取信息、加工处理信息以及创造新信息的能力。它 包括：信息工具的使用能力; 获取识别信息的能力; 加工处理信息的能力; 创造、传递新信息的能力。个 体应有在对所掌握的信息从新角度、深层次加工处理的基础上，进行信息创新，从而产生新的信息的能力。

\section{4 信息道德}

信息道德是指人们对信息进行获取、使用、创造和传播过程中应该遵守一定的道德伦理规范。具体表 现在：遵守信息技术的法律法规，文明上网; 不制造和传播计算机病毒，窃取他人信息; 不制造和传播黄 色、反动信息, 辱骂他人; 参与盗版活动, 尊重知识产权等 [4]。

\section{3 少数民族大学生信息素养现状}

本次调查以西北民族大学在校本科生作为调查对象。调查问卷针对民族高校大学生的信息素养、信息 知识、信息能力、信息道德等方面问题进行设计。问卷调查采用随机抽样的方法，现场发放并回收。共发 放问卷 300 份, 回收有效问卷 297 份, 有效问卷回收率为 99.00\%, 采用专门的 EXCEL 统计软件进行数据 分析。 


\section{1 信息意识现状}

表 1. 少数民族大学生信息意识现状

\begin{tabular}{ccc}
\hline 题目 & 选项 & 比例 \\
\hline 获取信息的目的 & 个人兴趣, 进行知识更新 & $73.8 \%$ \\
& 完成学业、配合学习需要 & $55.9 \%$ \\
& 休闲娱乐、增加谈资 & $37.9 \%$ \\
& 遇到问题、难题解惑 & $44.8 \%$ \\
\hline 整理获取信息的频率 & 其他 & $8.3 \%$ \\
& 一直会 & $21.4 \%$ \\
& 偶尔会 & $47.6 \%$ \\
& 视重要程度而定 & $25.5 \%$ \\
\hline 清楚与本专业相关的核心期刊 & 没有这方面意识 & $5.5 \%$ \\
& 非常清楚 & $2.1 \%$ \\
& 比较清楚 & $35.9 \%$ \\
& 不太清楚 & $55.2 \%$ \\
\hline 查找信息的主动性 & 从完全不清楚 & $6.9 \%$ \\
& 总能主动查找 & $25.5 \%$ \\
& 多数时候能主动能够查找 & $60.0 \%$ \\
& 有时能主动查找 & $13.8 \%$ \\
& 从动查找 & $0.7 \%$
\end{tabular}

调查结果如表 1 所示。少数民族大学生的信息意识比较高。他们明确获取信息目的, 对于信息的查找, $25.5 \%$ 的同学总能进行主动查找, $60.0 \%$ 的同学能在多数时候进行主动查找, 而从不主动查找的人数只占 $0.7 \%$; 对于获取信息的整理, $94.5 \%$ 的同学可以根据信息的价值, 对获取的信息进行不同程度的整理, 只 有 5. 5\%的同学没有这方面的意识, 对信息进行合理的整理, 方便对信息的使用及为下次的需要提供便捷利; 对于与本专业相关的核心期刊, $61.1 \%$ 的同学不太清楚或不清楚, 这将影响他们对本专业前沿知识的了解。

\section{2 信息知识现状}

表 2. 少数民族大学生的信息知识现状

\begin{tabular}{ccc}
\hline 题目 & 选项 & 比例 \\
\hline 你对 office 办公软件的了解及使用情况 & 很熟悉, 经常使用 & $75.3 \%$ \\
& 比较熟悉, 偶尔使用 & $22.6 \%$ \\
& 了解一点, 几乎不用 & $2.1 \%$ \\
& 不了解, 没用过 & $0 \%$ \\
\hline 你对互联网、报刊、电视、宣传册、光盘 & 非常熟悉 & $15.8 \%$ \\
等几种常见信息源的特点的了解情况 & 比较熟悉 & $30.2 \%$ \\
& 不太熟悉 & $36.7 \%$ \\
& 完全不知道 & $17.3 \%$ \\
\hline 你如何获取信息检索的有关知识 & 信息检索课 & $10.3 \%$ \\
& 信息检索讲座 & $3.5 \%$ \\
& 在互联网上 & $49.4 \%$
\end{tabular}


调查结果如表 2 所示。少数民族大学生的信息知识偏低。对于互联网、报刊、电视、宣传册、光盘等 几种常见信息源的特点的了解情况, 只有 $15.8 \%$ 的同学非常熟悉, 有 $17.3 \%$ 的同学完全不知道信息检索知 识。这将影响同学们在信息检索的过程中不能很好的选择信息源, 降低信息使用效率。对 office 办公软 件的了解及使用情况, $75.3 \%$ 的同学很熟悉且经常使用, 所有同学都使用 office 办公软件, 只是使用的频 率有所差距。通过办公软件使用, 可以帮助同学们将获取的信息内容进行整理和归类，提高信息的利用率。 对如何获取信息检索的有关知识, 只有 $10.3 \%$ 的同学参加过信息检索相关课程的学习, $3.5 \%$ 的同学听过有 关信息检索的讲座, $46.8 \%$ 的不知道信息检索的有关知识, 在平时的检索中只是凭感觉进行, 这使得检索 到有用信息的效率明显降低。

\section{3 信息能力现状}

表 3 民族高校大学生的信息能力现状

\begin{tabular}{ccc}
\hline 题目 & 选项 & 比例 \\
\hline 运用搜索到的信息 & 直接复制粘贴 & $24.8 \%$ \\
在原作基础上修改 & $44.1 \%$ \\
理解后用自己的话表达 & $30.3 \%$ \\
完全不能运用 & $0.7 \%$ \\
\hline 能否准确提取检索词 & 一直是 & $13.1 \%$ \\
& 经常 & $57.2 \%$ \\
& 有时 & $27.6 \%$ \\
清楚获取信息途径 & 从不 & $2.1 \%$ \\
\hline & 非常清楚 & $11.0 \%$ \\
& 比较清楚 & $65.5 \%$ \\
& 不太清楚 & $22.8 \%$ \\
& 完全不清楚 & $0.7 \%$ \\
\hline
\end{tabular}

调查结果如表 3 所示。少数民族大学生的信息能力比较低。在获取信息的途径方面, 有 $23.5 \%$ 的同学 不清楚获取信息的途径, 只有 $11.0 \%$ 的同学非常清楚信息获取的途径, 大多同学缺乏获取信息途径的能力; 在信息查找的过程中, 提取信息检索词的准确性, 70.3\%的同学都能够准确提取检索词, 但还有少部分同 学从不能准确提取检索词, 这样会严重影响信息查找的效率; 在信息的使用方面, 只有 $30.3 \%$ 的同学能够 在理解后用自己的话表达, $24.8 \%$ 的同学采用了直接复制粘贴的形式进行信息的使用, 同学们在使用信息 的过程中缺乏对其进行评价与分析, 严重降低了信息的利用率。 


\section{4 信息道德现状}

表 4 民族高校大学生的信息道德现状

\begin{tabular}{ccc}
\hline 题目 & 选项 & 比例 \\
\hline 对于传播的色情暴力内容 & 从来不看 & $17.20 \%$ \\
& 偶尔看 & $39.30 \%$ \\
& 不关心 & $25.50 \%$ \\
对盗版书籍光盘等所持态度 & 呼吁有关部门加以管理 & $17.90 \%$ \\
& 支持正版 & $13.10 \%$ \\
& 价格至上 & $51.00 \%$ \\
& 偶尔关心 & $30.30 \%$ \\
论坛发帖情况 & 从不关心 & $5.50 \%$ \\
\hline & 只发文明帖 & $54.50 \%$ \\
& 偶尔发文明帖 & $31.70 \%$ \\
& 从不发文明帖 & $8.30 \%$ \\
& 从不发帖 & $5.50 \%$ \\
\hline
\end{tabular}

调查结果如表 4 显示, 少数民族大学生的信息道德还比较薄弱。对于传播的色情暴力内容, 只有 $17.9 \%$ 的同学呼吁有关部门加以管理, $25.5 \%$ 的同学对此漠不关心, 其余的同学要么自己不看要么偶尔看看; 对 于盗版光盘书籍等, 有 $13.1 \%$ 的同学支持正版, 大多数同学认为价格至上, 只要价格合适, 就可以接受; 关于论坛发帖, 大多数人都能遵守网络道德, 只发文明帖, 而 $5.5 \%$ 的同学则习惯于发一些低俗的帖子, 甚 至发一些对他人进行人身攻击的帖子。这说明民族高校的大学生朋友们的信息免疫和甄别能力比较弱, 自 控、自律和自我调节能力都有待于进一步提高。

\section{4 提高少数民族大学生信息素养的措施}

\section{1 增加信息素养系列课程}

学校应该在学生刚进入大学时就开始开设信息素养的相关课程, 使大学生在第一时间接触信息素养, 信息意识。随着学生对专业课程学习的不断深入, 将信息素养教育与专业课程进行有机的结合, 让学生在 专业知识的基础上, 把获取信息的方法运用到专业课程的深入学习当中, 加强信息的获取能力, 增强信息 的处理能力, 提高信息的评价能力, 促进学生对信息的利用能力, 有意识、有目的、有计划地培养民族高 校大学生的信息素养。同时教师鼓励学生多参与科研活动, 提高民族高校大学生对信息的分析能力和创造 能力, 让学生在活动中获取一种成就感, 从而使民族高校大学生信息素养的培养得到良性的发展。

\section{2 提高教师的信息素养水平}

教师作为学生学习的引导者, 需要掌握一定的计算机网络知识及操作技能, 对于一些协助学习的软件, 教师要学会使用, 这样有利于教师在教学的过程中将这些信息传授给学生, 在教学的过程中, 教师尽量发 挥自己的主导作用, 在学习的过程中, 教师尽量引导学生发挥主体作用, 提高学生的信息能力 [5]。教师 除了自我提升信息素养外, 学校还应该给教师提供培训的机会, 是使教师的信息意识得到强化、信息知识 得到扩展、信息能力得到提高、信息道德得到加强, 在平时的教学过程中及时感知学生思维方式的新需求, 让学生在潜移默化中提高自身的信息素养 [6]。 


\section{3 加强图书馆资源使用体验}

信息素养源自于早期的图书馆检索，因此图书馆对大学生信息素养的培养有着得天独厚的优势。高校 图书馆有大量的信息资源, 同时也给同学们提供完善的信息检索工具, 方便同学们对信息素养培养方面的 实战演练。目前, 学校已开设文献检索课程, 但该课程只指定位于公共选修课, 能加入该课程学习的同学 都很有限, 若将该课程定位为必修课, 则每个同学都能加入到该课程的学习当中, 从而提高大学生的信息 知识, 促进信息意识、信息能力、信息道德等方面的提高 $[7]$ 。

\section{3.1 提高学生主动参与性}

高校图书馆拥有大量的信息资源, 且是一个免费的开放空间, 只要是本校学生, 都可以免费借阅图书 馆内的任何纸质资料, 西北民族大学图书馆的纸质文献拥有 150 万余册, 其中近 8 万册是清代以前的珍贵 文献; 只要是本校的 IP 地址, 都可以免费汶览本校的电子图书馆, 本校图书馆中拥有的电子文献 100 万 余册, 基本涵盖了学校的各个专业, 为教学和科研提供了强有力的信息支持。大学生朋友们应该主动地走 进图书馆，把它当作信息素养培养的实践场所，提高自身的信息素养。

\section{3.2 增加硬件和软件的投入}

提高图书馆对学生信息素养的培养需要完善的硬件支持, 加大硬件设施的投入, 可以使图书馆在信息 素养的培养方面发挥更大的作用。现代图书馆借助于网络通讯技术和多媒体超文本链接技术，可以为人们 提供远距离检索、远距离汶览等条件, 因此传统图书馆的功能向现代图书馆的功能发展不仅需要硬件设施 提供物质基础, 也需要信息技术提供软件基础 [8]。通过硬件设施与软件设备的结合, 图书馆可以将电子 馆藏接入校园网, 从而使学生足不出户也能汶览到图书馆内的电子资源, 提升自身信息素养。在西北民族 大学图书馆中, 拥有百兆主干计算机网络、 20 余台服务器、 $36 \mathrm{~TB}$ 海量存储、 40 余种数据库, 建成了专 用信息传递网络平台。图书馆向读者提供书刊目录、电子资源、馆际互借、参考咨询等信息的查询和链接 服务, 已初步形成多层次、全方位、开放式、高效率的文献信息服务体系, 为学校的教学和科研提供优质 高效的服务。学生可以根据自己的需要, 进行网上自主学习, 提高自身的信息素养。

\section{3.3 加强特色馆藏资源建设}

系统完整和专业的文献为学生在检索的过程中提供了便利, 增加了学生对检索的兴趣, 主动了解各类 学科的前沿知识, 吸收前人优秀的文化成果, 并将这些成果进行提取、加工、评价和组合, 提高驾驭信息 的能力, 形成自己的文化沉淀 [9]。学校图书馆应根据社会发展需求和信息发展需要, 通过各种途径引入 前沿书刊资料, 让同学们及时了解各种前沿信息, 从而对图书馆产生兴趣, 主动进入图书馆进行检索, 无 形中提高了自身的信息素养 [10]。

\section{3.4 对学生进行专门培训}

由于一些学生不关心图书馆的信息, 从而对图书馆的使用知之甚少, 图书馆馆员应该采取各种有效的 培训和教育途径, 提高学生对图书馆的认识、了解图书馆的使用, 使学生对信息资源的利用率得到提高。 在新生入学时, 举行入馆教育, 让学生了解图书馆的基本情况, 知道图书馆的资源和使用方法。面对不同 的专业开展专题培训, 从专业角度全面、系统地介绍本专业的各种文献资源和重要的检索工具。除此外, 图书馆馆员可策划开展活动, 如读书心得交流、图书推荐等形式让学生更加了解信息资源, 主动加入读书 行列, 提升自身的信息素养。 


\section{5 结语}

在信息化时代，一个人想立足于知识经济社会中，就离不开信息素养。提高信息检索、信息处理、 信息识别、信息评价、信息加工、信息传递和信息创造的能力是当代少数民族大学生的必备素质, 信息素 养教育必将成为民族高校创新人才培养的长期目标。培养少数民族大学生的信息素养已经成为民族高校教 育教学改革的重要课题之一 [11]。因此, 民族高校必须不断地培养和提高大学生的信息素养, 完善大学生 的知识结构, 强化学生的信息运用技能，全面提高大学生的整体素质。

\section{6 致谢}

此项研究受到以下项目支持 “西北民族大学 “一优三特” 学科 2017 年度中央高校基本科研业务费项 目 “西北民族研究””（N0：31920170096); “运用电子学档促进教学效果的研究” (N0：2014JG-2670030634)

\section{参考文献:}

[1] 李雅琴. 浅论大学生信息素养的培养 $[J]$. 山西青年管理干部学院学报, $2012(3) ： 23-27$.

[2] 钟志贤. 信息素养：培养你八大能力 $[\mathrm{J}]$. 中国教育报, 2014（3).

[3] 孙平，曾晓牧. 面向信息素养论纲 $[J]$ ． 图书馆论坛，2015(4)：44-49

[4] 李智晔. 大学生网络信息素养的培养机制与方法情报科学, 2015 (5)：122-126.

[5] 吕庆阳，刘孝文．国内外信息素质概念的界定河北科技图苑 [ J ]．2013(2)：76-80.

[6] 肖茜. 浅论普通高等学校学生信息素养的培养 $[\mathrm{J}]$. 苯庄学院学报, 2014 (8)：233-238.

[7] 韩冰融. 从现代大学生的信息素养看我国的信息素质教育 $[\mathrm{J}]$. 内蒙古电大学刊, 2012 (1)：144-148.

[8] 吴晓燕．浅谈高校学生信息素养的培养策略 [ J ］．上海青年管理干部学院学报，2013（1）：78-83.

[9] 王吉庆. 信息素养论[M]. 1. 上海教育出版社，1999：125-122.

[10］张厚生. 信息素养 [M]. 1. 南大学出版社, 2014：88-90.

[11] ANDERSON J R. ACT:A simple theory of complex cognition[J]. American Psychologist, 1996, 51 (4) : 355-365.

\section{References}

[1] Y.Q.Li: Discussion on the Cultivation of College Students' Information Literacy[J], Journal of Shanxi College For Youth Administrators, 2012(3) : 23-27.(In Chinese)

[2] Z.X.Zhong: Information Literacy: The Cultivation of Your Eight Abilities[J], China Education Daily, 2014(3).(In Chinese)

[3] P.Sun and X.M.Zeng: Towards Information Literary[J], Library Tribune, 2015(4) : 44-49(In Chinese)

[4] .Y.Li: The Training Mechanism and Methods of College Students' Network Information Literacy, Information Science, 2015(5): 122-126.(In Chinese)

[5] Q.Y.Lv and X.W.Liu: Definition of Information Literacy at Home and Abroad[J], Hebei Sci-tech Library Journal, 2013(2) : 76-80.(In Chinese)

[6] Q.Xiao: Discussion on the Training of Students' Information Literacy in Ordinary Colleges and Universities[J], Journal of Zaozhuang University, 2014(8): 233-238.(In Chinese)

[7] B.R.Han: Looking at the Information Literacy Education in China from the Information Literacy of Modern College Students[J], Journal of Inner Mongolia Radio\&TV University, 2012(1): 144-148.(In Chinese)

[8] X.Y.Wu: Discussion on the Training Strategy of Information Literacy of College Students[J], Journal of Shanghai College for Youth Administrators, 2013(1) : 78-83.(In Chinese)

[9] J.Q.Wang: Information Literacy Theory[M], Shanghai Education Publishing House, 1999: 125-122.(In Chinese)

[10] H.S.Zhang: Information Literacy[M], Southeast University Press, 2014: 88-90.(In Chinese)

[11] ANDERSON J R.ACT:A Simple Theory of Complex Cognition[J], American Psychologist,1996, 51(4): 355-365.(In Chinese) 